\title{
Molecular Hydrodynamics of High Explosives
}

\author{
RECEIVED \\ APR 121996 \\ OSTI
}

James Belak

November 1994

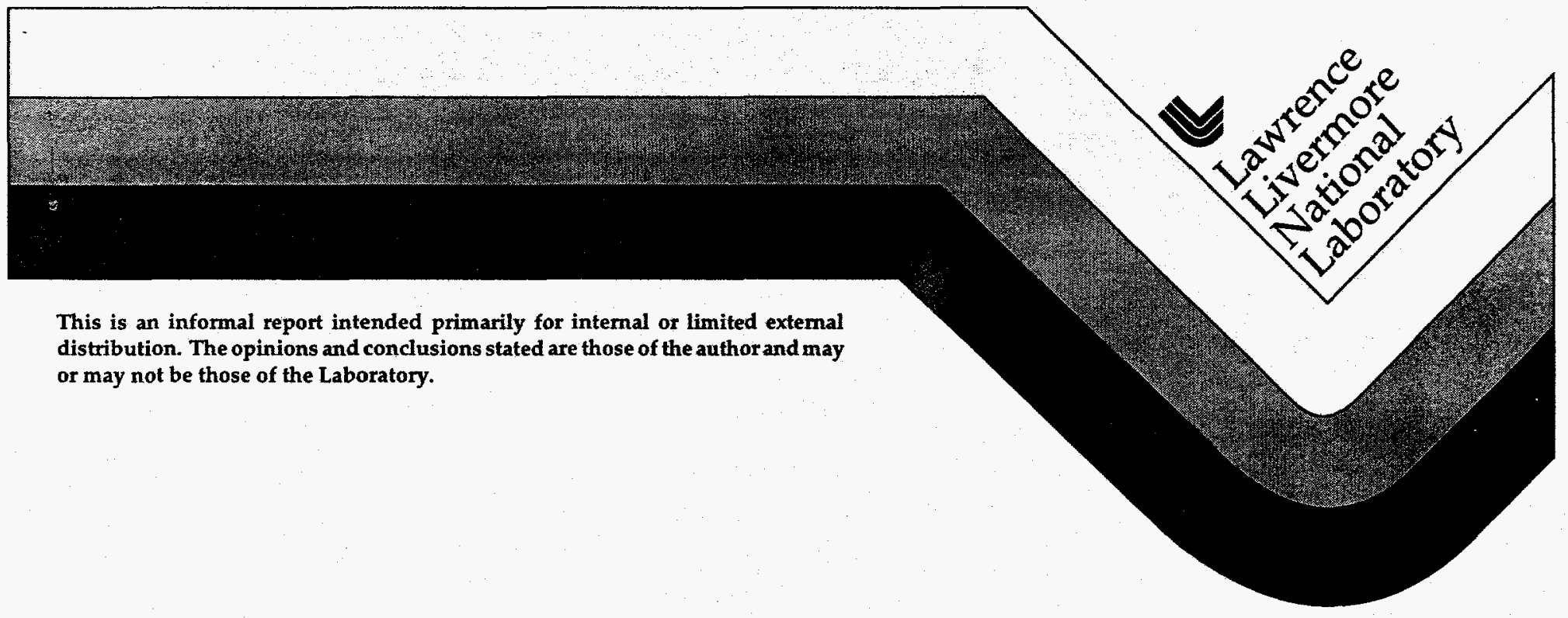




\section{DISCLAIMER}

This document was prepared as an account of work sponsored by an agency of the United States Government. Neither the United States Government nor the University of California nor any of their employees, makes any warranty, express or implied, or assumes any legal liability or responsibility for the accuracy, completeness, or usefulness of any information, apparatus, product, or process disclosed, or represents that its use would not infringe privately owned rights. Reference herein to any specific commercial product, process, or service by trade name, trademark, manufacturer, or otherwise, does not necessarily constitute or imply its endorsement, recommendation, or favoring by the United States Government or the University of California. The views and opinions of authors expressed herein do not necessarily state or reflect those of the United States Government or the University of California, and shall not be used for advertising or product endorsement purposes. 


\section{Molecular Hydrodynamics of High Explosives}

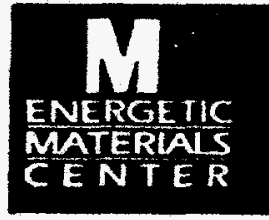

James Belak

University of California, Lawrence Livermore National Laboratory, Livermore, CA 94550

\begin{abstract}
Motivation
High explosives release mechanical energy through chemical reactions. Applications of high explosities are rast in the mining and military industries and are theginning to see more civilian applications such as the deployment of airhags in modern automobiles.

One of the central issues surrounding explosive materials is decreasing their sensitivity. necessary for their sate handling. while maintaining a high vield. Many practical tests have been devised to Jelermine the sensitivity of explosive materials to shock. to impact. to spark. and to triction. These lests have greal value in determining yield and setting precautions for safe handling but tell little of the mechanisms of initiation. How is the mechanical energy of impact or friction transformed into the chemical excitation that initiates explosion? The answer is intimately related to the structure of the explositi material. the sia and distribution of grains. the size and presence of open areas such as bids and gas bubless. and ineritably the bonding between explusive mulecules.
\end{abstract}

\section{What is a high explosive?}

Explosive materials. like HMX and TATB. are composed of relatively large lloppy molecules with many inlernal modes of viblation. The molecule is in a mitulathe stale from which an enormous energy is released during detonation.
Initiation occurs by lowering the barrier between the metastable and decomposed states. There is evidence that, for the majority of explosives. this initiation may be thermal in nature. The mechanical energy is degraded into heat and concentrated into a small region to torm a hot spot. This heat then enters the molecule through internal vibrational modes that are strongly coupled to the lattice vibrations. effectively lowering the barrier to decomposition. The response due to a strong shock may be somewhat different. The collisions between molecules at a strong shock front are so great that the non-equilibrium motion of the shock preferentially couples to specitic internal vibrations of the molecules. It is these vibrational modes. which may be different than the modes that strongly couple to lattice vibrations, through

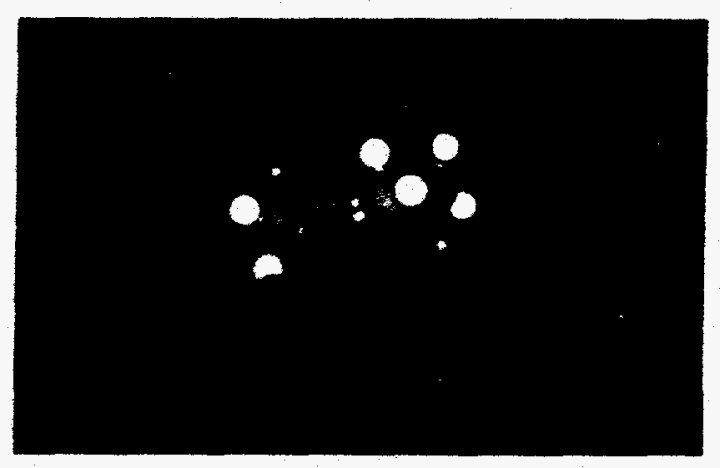

Figure 1. A computer rendition of an HMX molecule showing the bonding between the atoms. The gray atoms are carbon. the blue are nitrogen, the red are oxygen, and the white are hydrogen. 


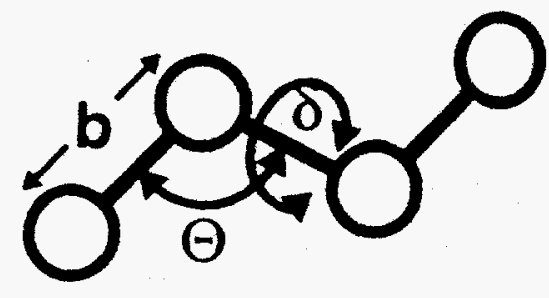

Figure 2. The internal degrees of freedom of a tloppy molecule. These include the length of bonds. the angle between three atoms that are bonded, and the rotation about the center bond when four atoms are bonded.

which energy enters the molecule at a strong shock front.

\section{How do we model it on a computer?}

The molecular dynamics (MD) computer simulation method is an ideal approach to study energy transport during shock loading and related problems such as the reactive dynamics of chemically sustained shocks. Within the MD approach. the response of a material to an impact is evaluated by following the motion of every atom in the material. The force on each atom is computed and the motion is simulated by solving the classical equations of motion ( $\mathrm{F}=\mathrm{ma}$ ).

There are two types of forces that determine the motion of energetic molecules. The first type is the strong internal covalent bonds. These are the forces that determine the geometry of the molecule. A model for these forces in terms of the length of bonds. the angle hetween three atoms that are honded. and the rotation about the center bond when four atoms are bonded (see Figure 2) has been developed within the biochemistry community to study Hoppy protein molecules. The other type of force needed to simulate energetic molecules is the forces between the molecules. This includes the strong repulsive forces that delermine the sise of the molecule and hing ranged coulemb firces. The long ranged lorees arise

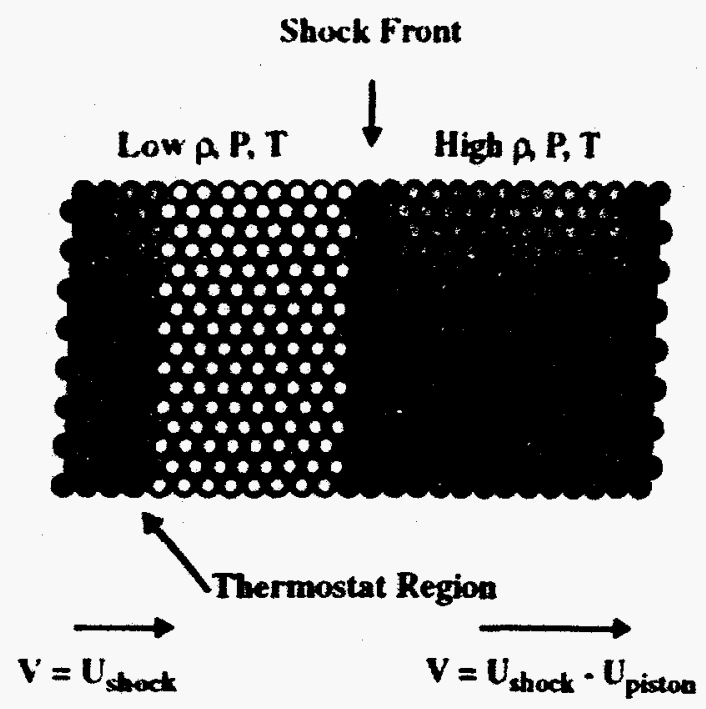

Figure 3. A schematic illustration of the boundary conditions used to simulate shock propagation within the reference frame of the shock. The molecules flow from left to right in the figure and the shock front is stationary.

because covalent bonds involve the sharing of electrons between atoms and in some of these bonds the electrons spend more time around one atom than another. This separation of electronic charge leads $w$ the long ranged force. A model of this type has been constructed for HMX at LLNL by Larry Fried.

There are many ways to simulate the propagation of a shock using molecular dynamics methods: in the material reference frame by driving a fixed wall (piston) into the material or in the piston reference frame by drive the material into a stational wall. For our purposes, however. we want to understand the hehavior of the explosive molecules at the shock front. To do this we have devised a special boundary condition in which the shock front is stationary and we are able to study the shock propagation for arbitrarily long periods of time. Molecules are continually inserted into the simulation eell from the left at a constant velocity (L, inew and are removed on the right at a slower constant velocity $\left(\mathcal{L}_{\text {sheck }}-\mathcal{L}_{\text {piston }}\right)$. The 


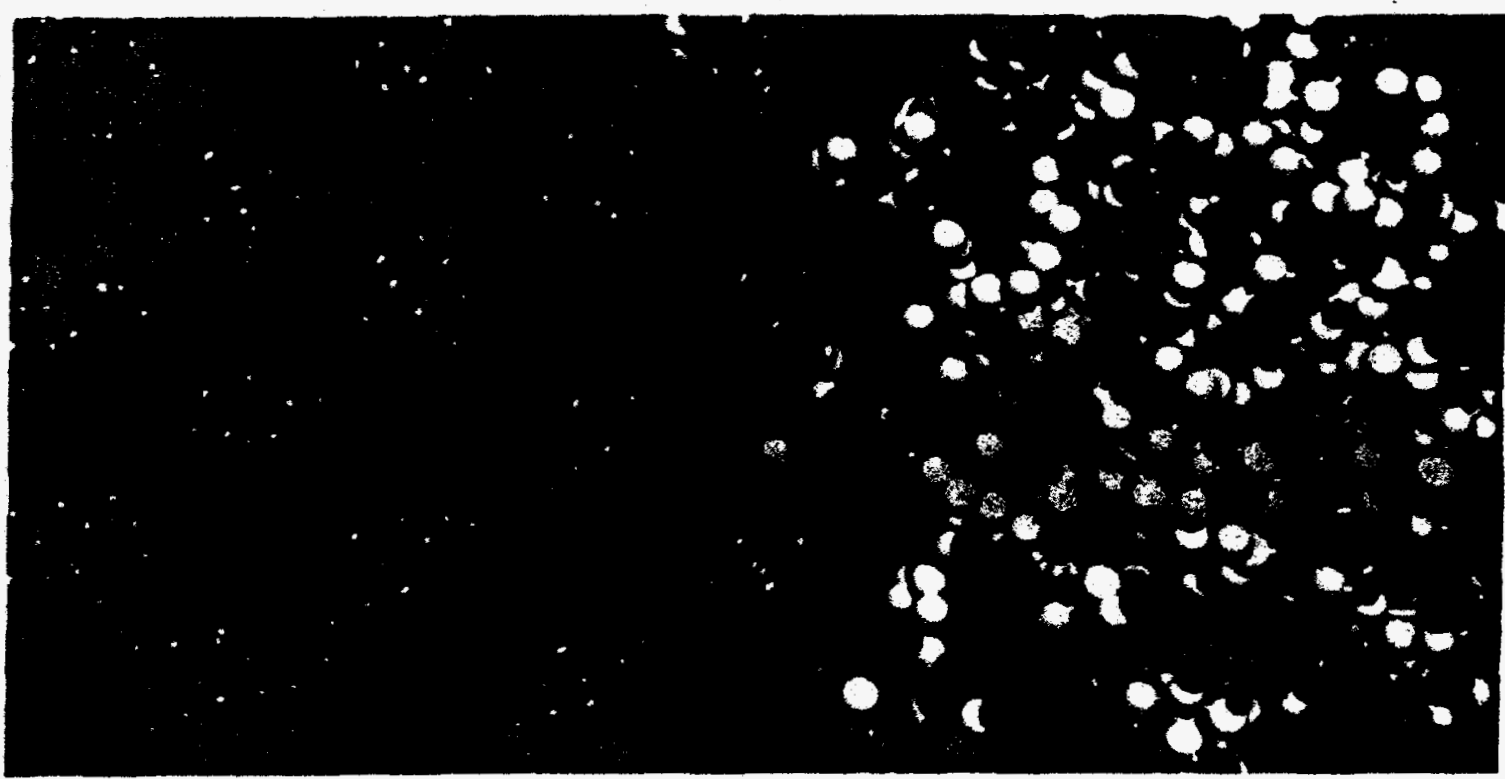

Figure 4. A molecular dynamics computer simulation of a shock wave propagating in the high explosive HMX. The atoms shaded blue in the low density region on the left are at room temperature while the atoms shaded green and red in the high density region on the right are very hot (several thousand Kelvin).

result is that material piles up at the departing boundary and a density discontinuity propagates into the simulation cell (to the left in Figure 3). This density discontinuity is the shock front.

\section{What can we learn?}

The method described above has been used to simulate shock propagation in simple atomic solids, including argon and copper, small molecular systems, and most recently high explosives. The simulations give calculated shock speeds (Hugoniot relations) in excellent agreement with experimental measurements. Shown in Figure 4 is a molecular dynamics computer simulation of a shock wave propagating in the high explosive HMX. The atoms shaded blue in the low density region on the left are at room temperature while the atoms shaded green and red in the high density region on the right are very hot. Our calculated temperature at the shock front for this $40 \mathrm{GPa}$ shock exceeds 10,000 Kelvin! This energy. however. at the shock front is only in the center of mass motion, the motion of the molecule as a whole. HMX has a large number of internal vibrational modes (about 80) and very quickly the energy from the shock dumps into the internal vibrations. In our simulation this process takes place within a few picoseconds or within a few nanometers behind the shock front (a distance comparable to a few molecular diameters). The temperature far behind the shock is much less, about 1000 Kelvin.

As mentioned above, much of the sensitivity of high explosive materials is thought to be determined by the material microstructure. This is based upon the experimental observation that perfect crystals are extremely difficult to detonate while crystals with lots of defects detonate easily. The types of defects include the boundary between the explosive material and its binder, grain boundaries between crystalline regions with different orientations, the presence of dislocations, and the presence of large open regions (voids) that may contain gas molecules. The internal fiction energy when a dislocation moves generates local heat as 
does the PV work when a large void is compressed.

The advent of large massively parallel supercomputers enables a unique opportunity to examine the role of microstructure in determining sensitivity with atomic-scale detail. On a present generation massively parallel supercomputer we can store all of the information required to simulate the motion of hundreds of millions of atoms (or millions of HMX molecules). This represents a region of space greater than 100 nanometers on a side, comparable to the large voids of interest. However, because of the enormous computational work required to perform such a simulation, timescales of no more than a few picoseconds are currently possible for these large systems. Presently we are limiting our massively parallel supercomputer simulations to a few tens of thousands of HMX molecules, or void sizes that are no more than a few nanometers across.

We simulate the propagation of a shock wave through a void by removing a tew molecules in a small region in front of the shock. Because of the nature of our simulation (periodic boundary conditions within the plane of the shock) we are effectively simulating shock propagation through a very high density of small voids. We find something interesting. The equilibrium temperature, far behind the shock, increases quite dramatically with increasing void size. This is shown in Figure 5 for an $80 \mathrm{GPa}$ shock and void sizes up to $10 \mathrm{~mol}$ ecules in HMX.

Recently, we have simulated a dislocation in HMX and the behavior of the molecules near the dislocation edge. These molecules are strained and we expected them to be more easily excited at a shock front.

\section{Where do we go from here?}

Molecular dynamics simulations give an enormous amount of detail concerning processes like shock propagation and energy transport. but are

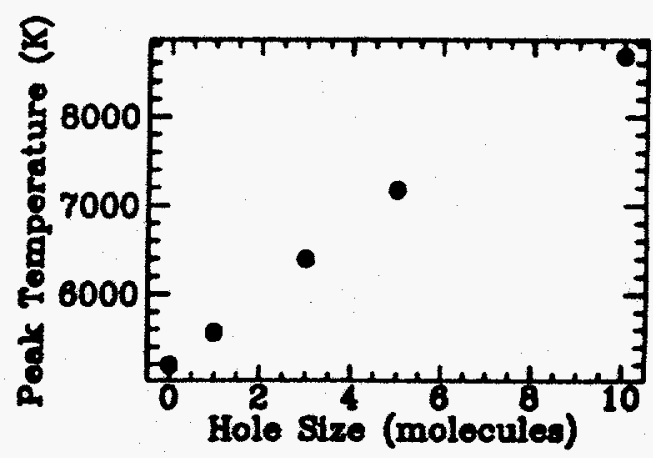

Figure 5. Peak temperature at the position of a compressed void a few nanometers behind the shock front for an $80 \mathrm{GPa}$ shock in HMX.

limited in solving practical engineering problems because of the enormous amount of work involved. We need a mechanism to take the atomic-scale information we learn from the MD simulations and translate it to practical information that can be used on the macroscopic scale. One method is to develop phenomenological models of materials behavior, like the constitutive models used in continuum mechanics. These models keep track of the material microstructure (the voids, grains, dislocations, and so on) but not the individual atoms and molecules. Our goal is to use MD simulations to guide the development of better phenomenological models and determine their parameters from a firm science base.

\section{Acknowledgments}

This work would not have been possible without numerous discussions with Clark Souers, Larry Fried, Al Nichols, and Craig Tarver and was performed at Lawrence Livermore National Laboratory under the auspices of the U.S. DOE through Contract No. W-7405-ENG-48. 


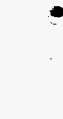

